\title{
$\bullet \cdot$ Sulforaphane and Its Relationship with the Intestinal Flora
}

IJCRR

Section: Healthcare

Sci. Journal Impact

Factor: 5.385 (2017)

ICV: 71.54 (2015)

\section{Pooja Dosieah1, Tan Luxuan², Zhang Zhenyu ${ }^{3 *}$}

\section{ABSTRACT}

Sulforaphane is a compound that is correlated to the iso-thiocyanate family.It is well studied to be anti-carcinogenic preventing stomach cancers, antimicrobial, anti-inflammatory and neuroprotective in addition to its sufficient ability to protect against aging and diabetes. Sulforaphane reveals a direct positive effect on the intestinal tissues as it shows a sufficient ability in presenting a high antimicrobial respond that leads to the prevention of uprising various bacteria including both the gram-positive and the gram-negative ones such as Escheria coli and Helicobacter pylori in addition to other types of bacteria. The main objective of this study is to report a profound review of the recent papers done on this topic in order to clarify the relationship between the sulforaphane and intestine. For this review, however, there are no many articles that indicate the intimate relation, ten articles and reports from PubMed and Google Scholar databases have been included in this review of literature in order to demonstrate sulforaphane and its functions and effects towards the intestinal flora. This updated literature synthesis compromised that sulforaphane has a direct positive influence on the intestinal flora. Thus more attention should be put into consideration regarding the increase of its intake.

Key Words: Sulforaphane, Broccoli sprouts, Myrosinase enzyme, Glucoraphanin, Intestinal flora

\section{INTRODUCTION}

Sulforaphane is simply obtained when the enzyme myrosinase is capable of transforming the glucosinolate glucoraphanin into sulforaphane [1]. It is well known that the enzymes glucoraphanin and myrosinase are located in different sites of the plant anatomy, this mechanism change takes place when the plant is prone to damage may be through different ways as an example chewing, blending and chopping allowing the bi-product of the compounds to be mixed and reacted [2]. The numerous small aged broccoli sprouts, as well as cauliflower, are considered to be a rich reservoir of the enzyme good glucoraphanin. Amongst, sulforaphane is also a section from an entire chain of disease preventions phytochemicals through plants, which are known as called isothiocyanates [3]. In the body, sulforaphane works as a stimulator of basic enzymes secretion, which is capable of neutralizing the free radicals [4]. Although inflammation in addition to free radicals is considered to be causes of different types of malignancy. Thus, Isothiocyanates can also function as cancer activating enzymes blocker in the human body system, leading to the second way of prevention [5]. Sulforaphane has been reported as basic offensive tool towards the infec- tions through Helicobacter pylori bacteria, which are highly correlated within the global pandemic of gastrointestinal malignancy and it is a strong catalysing way of protective enzymes production that is capable of preventing inflammation and oxidation through the transcription factor Nrf2 (NF-E2 p45-related factor-2; that also can attack $\mathrm{H}$. pylori in vitro and it also inhibits the formation of stomach tumours that are chemically induced in a rodent model [6].

\section{Sulforaphane Composition and its function}

The Cellular composition of Broccoli and other several leaves has been reported for many years. Several studies have been done on this part and it was concluded that most of the broccoli sprouts contain massive amounts of vitamins and minerals due to the presence of glucosinolates, which are healthy for the human body intake [7,8]. According to the recent statistics done, it has been demonstrated that the components of broccoli seed mainly the glucoraphanin which is existent in high amounts in addition to the sulforaphane and isothiocyanate which a baseline of cancer attack. Sulforaphane as one of the components of the broccoli sprouts is presented in several cruciferous vegetables. It has been

\section{Corresponding Author:}

Zhang Zhenyu, Department of Gastroenterology, Affiliated Hospital of Nanjing Medical University (Nanjing First Hospital), Nanjing, Jiangsu 210006, China; Ph: +8618951670222; Email: zhangzhenyu808@126.com

ISSN: 2231-2196 (Print)

Received: 27.08 .2018
ISSN: 0975-5241 (Online)

Revised: 12.09 .2018
Accepted: 29.09 .2018 
revealed for its ability to induce phase II detoxification enzymes and considered to be an anti-carcinogenic. Further studies have been suggested as another challenging function of sulforaphane in correlation with the chemoprotectivemaneuver dealing with the direct termination of the activity of HDAC (Histone deacetylase)[9]. The latent has been accomplished through the recent studies on the human colon malignant cells as well as human prostate cells, resulting in an excessive histone acetylation has been reported on the exposure sulforaphane [10]. The available results of HDAC inhibition via sulforaphane are correlated with malignant cells of the breast. Sulforaphane has the ability to reduce the xenografts of the prostate cancer in addition to its ability to suppress the intestinal polyps according to the experiment done on mice with an association with altered histone acetylation and the inhibition process of HDAC. Another study has presented that HDAC could be inhibited by the sulforaphane $\mathrm{N}$-acetyl cysteine after metabolism, other studies have been illustrated the influences of the HDAC inhibition on the isothiocyanates. Synthetic isothiocyanate phenylhexyl is capable of accomplishing the inhibition of HDAC and the chromatin and their role in leukemia that stops the rate of growth [11]. According to a study performed on the inhibition of the HDAC and it was concluded that there is a correlation within the histone marks. Although there is a slight increase in the amount of the intake in the histone that underwent acetylation as $\mathrm{H} 3$ and $\mathrm{H} 4$ as well as $\mathrm{H} 3 \mathrm{~K} 4$ and $\mathrm{H} 3 \mathrm{~K} 9$ after methylation in coordination with the histone loss. [12]. It has been concluded that the intake of sixty-eight grams of broccoli sprouts could be able to inhibit the HDAC via the peripheral blood mononuclear cells with the time ranges from three to six hours in addition to the histone induction of acetylated $\mathrm{H} 3$ and $\mathrm{H} 4$ [13]. The mentioned results could be a reference to provide the transitional approach of the HDAC inhibition through natural diets intake as broccoli sprouts [14].

\section{Sulforaphane and its anti-carcinogenic effect}

Nowadays, Cancer is considered to be a critical disease that leads to approximately $13 \%$ of the rate of the mortality globally which is increasing rapidly within the recent years [15]. Many causes could lead to cancer, one of them is related with the Life routine especially for the inappropriate nutrition and the bad habits associated with it which can be aetiologies for cancer, thus individuals should put into their consideration how important is the proper diet intake and avoid those health threatening lifestyles [16]. Healthy suggestions on the daily diets intake should be systemized with correlation with therapies in order to make a basic defence block against different kinds of diseases, it could also be achieved by adding some Hippocratic as well as traditional Chinese medicine [17]. The positive outcome of the diets intake based on plants was associated with the ability to be anti-cancerous and antioxidant in addition to the stoppage of some enzymes that could cause malignancy $[18,19]$. Several types of research have been done on this approach and it has been reported the proper daily diets that depend on the plant can protect against lung cancer, and some other body systems mainly the digestive system as an example stomach cancer and oesophageal cancer in addition to oral cancers [20]. Normally, for any human diet, its components mainly contain substances responsible for pleiotropic action mechanisms that in term impact the multiple targets in both intracellular and extracellular complex. The mentioned reaction is a big beneficial mechanism to provide protection against cancer. It is highly recommended to use sulforaphane as a main ingredient in the human daily diets because of its ability to be anti-inflammatory, antioxidant and anti-carcinogenic [21].

As reported by the research team of the experimental laboratory regarding the gastrointestinal tract, Sulforaphane phase two detoxification enzymatic activity, undergoing mediation process through ARE-NRF2 pathway as an example the enzyme glutathione transferase, UDP-glucuronyltransferase, NAD (P) H:quinoneoxidoreductase I and hemi-oxygenase-1 (HO-1), that leads to an opposite influence on the electrophilic and oxidative toxic products that needed to be diminished and inactivated prior to the macromolecular. Sulforaphane was also reported as a catalyst of interaction with KEAP1 by binding covalently to thiol groups of the inhibited proteins. Furthermore, the process of the expression of genes by the oligonucleotide implied a function of sulforaphane for the up-regulation expression of the NQO1, GST and GCL that found in the small intestine of experimental mice of the wild type [22]. However, the other type of mice Nrf2-null mice showed a decreased of enzymes secretion. Cornell at et al revealed the Nrf2 breakdown with siRNA association with sulforaphane induction hemi-oxygenase-1 (HO-1) upregulation $[23,24,25]$. Similar related impacts have been approached in other various studies correlated with vivo and it has been concluded that sulforaphane shows a high tendency to be a direct cause of phase two enzyme in experimental under the sulforaphane intake for several days with high amounts that may reach $1000 \mathrm{mml} / \mathrm{kg}[26,27,28]$. A critical issue that should be put into consideration indicates the ROS depletion through the usage NRF2 as an agent containing sulforaphane to be able to stop the initiation of being cancerous to the healthy tissues, Even though the activation of the NRF2 pathway in the final step could be affected by the sufficient mechanism of the specific treatments including the chemotherapies and the radiotherapies that mainly depend on ROS production [29,30,31].

\section{CONCLUSION}

Sulforaphane is a constituent of cruciferous vegetables; its existence in a human diet leads to a healthy environment for the body system as Sulforaphane is highly antioxidant to the adverse effects of the toxic substances in addition to 
its ability of being anti-carcinogenic, it was also found that sulforaphane is capable of preventing cellular inflammations and pain because of the high rate of the enzyme glutathione induction and it is also able to prevent cancer cells from entering the breast ability not only for the cardiothoracic system but also for other systems indicating prostate cancers liver and others, recent researches have been revealed a direct correlation between the sulforaphane within the intestinal flora in the digestion process, that enhances the entire process of metabolism as the mechanism of its interaction is initiated the reaction within the human body mainly the area of interest which is located at the gastrointestinal tract, that leads to the production of some enzymes that are defensive to damage via any kind of chemicals which in coordination protects the gastric cells to be, and an optimum prevention of gastric atrophy, in addition, it was also reported that Sulforaphane reveals a critical issue in the preventing H-pylori and capable to help for protection against various types of cancer including stomach cancer. Thus, studies on sulforaphane are not sufficient to know the long-term effects, some show, no adverse effects are reported within the high dose intake but other studies have been revealed a negative impact regarding the large amounts of sulforaphane in the daily intake, so further studies are highly recommended to figure out the effect of the large doses in the human body systems.

\section{ACKNOWLEDGEMENT}

I would like to extend my gratitude to my professor, Dr Zhang Zhen Yu and my colleague Dr Tan Lu Xuan for encouraging me and helping me in writing this article.

I am also grateful to all the authors, editors and publishers of all those articles, journals and books from where the literature for this article has been reviewed and discussed.

Source of Funding: No funding

Conflict of Interest: No conflict of interest.

\section{REFERENCES}

1. Nakamura K, Koyama M, Ishida R, Kitahara T, Nakajima T, Aoyama T. Characterization of bioactive agents in five types of marketed sprouts and comparison of their antihypertensive, antihyperlipidemic, and antidiabetic effects in fructose-loaded SHRs. J Food Sci Technol. 2016;53(1):581-90.

2. Senanayake GV, Banigesh A, Wu L, Lee P, Juurlink BH. The dietary phase 2 protein inducer sulforaphane can normalize the kidney epigenome and improve blood pressure in hypertensive rats. Am J Hypertens. 2012;25(2):229-35.

3. Talalay P, Fahey JW, Healy ZR, et al. Sulforaphane mobilizes cellular defenses that protect skin against damage by UV radiation. Proceedings of the National Academy of Sciences of the United States of America. 2007;104(44):17500-17505. doi:10.1073/pnas.0708710104.
4. Tortorella SM, Royce SG, Licciardi PV, Karagiannis TC. Dietary Sulforaphane in Cancer Chemoprevention: The Role of Epigenetic Regulation and HDAC Inhibition. Antioxidants \& Redox Signaling. 2015;22(16):1382-1424. doi:10.1089/ars.2014.6097.

5. Abbaoui B, Riedl KM, Ralston RA, et al. Inhibition of Bladder Cancer by Broccoli Isothiocyanates Sulforaphane and Erucin: Characterization, Metabolism and Interconversion. Molecular nutrition \& food research. 2012;56(11):10.1002/ mnfr.201200276. doi:10.1002/mnfr.201200276.

6. Royston KJ, Tollefsbol TO. The Epigenetic Impact of Cruciferous Vegetables on Cancer Prevention.Current pharmacology reports. 2015;1(1):46-51. doi:10.1007/s40495-014-0003-9.

7. Cheung KL, Khor TO, Kong AN. Synergistic effect of combination of phenethyl isothiocyanate and sulforaphane or curcumin and sulforaphane in the inhibition of inflammation. Pharm Res. 2009;26(1):224-31.

8. Clarke JD, Hsu A, Riedl K, et al. Bioavailability and inter-conversion of sulforaphane and erucin in human subjects consuming broccoli sprouts or broccoli supplement in a cross-over study design. Pharmacol Res. 2011;64(5):456-63.

9. Shapiro T, Fahey J, Wade K, Stephenson K and Talalay P. Chemoprotective Glucosinolates and Isothiocyanates of Broccoli Sprouts. Cancer Epidemiol Biomarkers Prev. May 1 2001;10(5):501-508.

10. Sasaki M, Shinozaki S, Shimokado K. Sulforaphane promotes murine hair growth by accelerating the degradation of dihydrotestosterone. BiochemBiophys Res Commun. 2016;472(1):250-4.

11. Gan N et al. "Sulforaphane Activates Heat Shock Response and Enhances Proteasome Activity through Up-Regulation of Hsp27." The Journal of Biological Chemistry 285.46 (2010): 35528-35536. PMC. Web. 12 Mar. 2018.

12. Kikuchi M et al. "Sulforaphane-Rich Broccoli Sprout Extract Improves Hepatic Abnormalities in Male Subjects." World Journal of Gastroenterology 21.43 (2015): 12457-12467. PMC. Web. 12 Mar. 2018.

13. Baier, Scott R. et al. "Off Target Effects of Sulforaphane Include the de-Repression of Long-Terminal Repeats through Histone Acetylation Events." The Journal of Nutritional Biochemistry 25.6 (2014): 665-668. PMC. Web. 13 Mar. 2018.

14. Kanazawa University. "Sulforaphane, a phytochemical in broccoli sprouts, ameliorates obesity." Science Daily, 7 March 2017. www.sciencedaily.com/releases/2017/03/170307100402.htm

15. Herr, I.; Buchler, M.W. Dietary constituents of broccoli and other cruciferous vegetables: Implications for prevention and therapy of cancer. Cancer Treat. Rev. 2010, 36, 377-383. 16.Moreno, D.A.; Carvajal, M.; López-Berenguer, C.; GarcíaViguera, C. Chemical and biological characterisation of nutraceutical compounds of broccoli. J. Pharm. Biomed. Anal. 2006, 41, 1508-1522.

16. Singh, S.V.; Singh, K. Cancer chemoprevention with dietary isothiocyanates mature for clinical translational research. Carcinogenesis 2012, 33, 1833-1842.

17. Wattenberg, L.W. Inhibition of carcinogenic effects of polycyclic hydrocarbons by benzyl isothiocyanate and related compounds. J. Natl. Cancer Inst. 1977, 58, 395-398.

18. Fahey, J.W.; Zalcmann, A.T.; Talalay, P. The chemical diversity and distribution of glucosinolates and isothiocyanates among plants. Phytochemistry 2001, 56, 5-51.

19. Shapiro, T.A.; Fahey, J.W.; Wade, K.L.; Stephenson, K.K.; Talalay, P. Human metabolism and excretion of cancer chemoprotective glucosinolates and isothiocyanates of cruciferous vegetables. Cancer Epidemiol. Biomark. Prev. 1998, 7, 1091-1100.

20. Zhang, Y.; Tang, L. Discovery and development of sulforaphane as a cancer chemopreventive phytochemical. Acta Pharmacol. 
Sin. 2007, 28, 1343-1354. Medicines 2015, 2152

21. Myzak, M.C.; Karplus, P.A.; Chung, F.L.; Dashwood, R.H.Anovel mechanism of chemoprotection by sulphoraphane:inhibition of histone deacetylase. Cancer Res. 2004, 64, 5767-5774.

22. Cornblatt, B.S.; Ye, L.; Dinkova-Kostova, A.T.; Erb, M.; Fahey, J.W.; Singh, N.K.; Chen, M.S.; Stierer, T.; Garrett-Mayer, E.; Argani, P.; et al. Preclinical and clinical evaluation of sulphoraphane for chemoprevention in the breast. Carcinogenesis 2007, 28, 1485-1490.

23. Myzak, M.C.; Tong, P.; Dashwood, W.M.; Dashwood, R.H.; Ho, E. Sulphoraphane retards the growth of human PC-3 xenografts and inhibits HDAC activity in human subjects. Exp. Biol. Med. 2007, 232, 227-234.

24. Zeng, H.; Trujillo, O.N.; Moyer, M.P.; Botnen, J.H. Prolonged sulforaphane treatment activates survival signaling in nontumorigenic NCM460 colon cells but apoptotic signaling in tumorigenic HCT116 colon cells. Nutr. Cancer 2011, 63, 248-255.

25. Gorrini, C.; Harris, I.S.; Mak, T.W. Modulation of oxidative stress as an anticancer strategy. Nat. Rev. Drug Discov. 2013, $12,931-947$.

26. Janssen-Heininger, Y.M.; Mossman, B.T.; Heintz, N.H.; Forman, H.J.; Kalyanaraman, B.; Finkel, T.; Stamler, J.S.; Rhee,
S.G.; van der Vliet, A. Redox-based regulation of signal transduction: Principles, pitfalls, and promises. Free Radic. Biol. Med. 2008, 45, 1-17.

27. Sporn, M.B.; Liby, K.T. NRF2 and cancer: The good, the bad and the importance of context. Nat. Rev. Cancer 2012, 12, 564-571.

28. Lee, J.S.; Surh, Y.J. Nrf2 as a novel molecular target for chemoprevention. Cancer Lett. 2005, 224, 171-184.

29. Taguchi, K.; Motohashi, H.; Yamamoto, M. Molecular mechanisms of the Keap1-Nrf2 pathway in stress response and cancer evolution. Genes Cells 2011, 16, 123-140.

30. Misiewicz, I.; Skupińska, K.; Kowalska, E.; Lubiński, J.; Kasprzycka-Guttman, T. Sulforaphane-mediated induction of a phase 2 detoxifying enzyme NAD(P)H:quinonereductase and apoptosis in human lymphoblastoid cells. Acta Biochem. Pol. 2004, 51, $711-721$

31. Dinkova-Kostova, A.T.; Holtzclaw, W.D.; Cole, R.N.; Itoh, K.; Wakabayashi, N.; Katoh, Y.; Yamamoto, M.; Talalay, P. Direct evidence that sulfhydryl groups of Keap1 are the sensors regulating induction of phase 2 enzymes that protect against carcinogens and oxidants. Proc. Natl. Acad. Sci. USA 2002, 99, 11908-11913. 